\title{
Entrepreneurial Projects Selection Problem Based on Stochastic Multi-attribute Acceptability Analysis

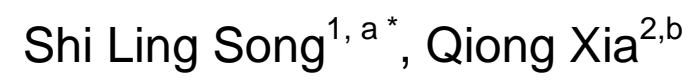

\author{
${ }^{1}$ School of Management, University of Science and Technology of China, 96 Jinzhai Road, Hefei \\ 230026, An Hui, P.R. China. \\ ${ }^{2}$ School of Economics, Hefei University of Technology,193 Tunxi Road, Hefei 230009, An Hui, P.R. \\ China.

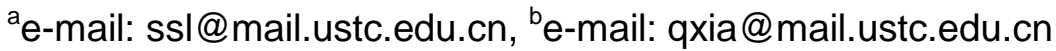

Keywords: Entrepreneurship; Project Selection; Multi-attribute Decision Making; SMAA.

\begin{abstract}
Selecting an entrepreneurial project is the first procedure to establish your business, and also one of the most significant procedure. However, it is not an easy task for the entrepreneurs to choose the most optimal entrepreneurial project from many attractive entrepreneurial projects. For one reason, they should inspect many criteria of the alternative entrepreneurial project. For another reason, the relative importance of different evaluation criteria is difficult to know. Prior studies haven't fully characterized the problem. In this paper we proposed a four-step approach based on Stochastic Multi-attribute Acceptability Analysis (SMAA) which aims to fill this gap.
\end{abstract}

\section{Introduction}

Nowadays, entrepreneurship is a popular word. More and more people want to start their own business. In china, as the fast development of the economy, the country has been a kingdom full of opportunities and challenges. More and more entrepreneurial myth created by the ordinary civilians lead to increasing hot wave of entrepreneurship.

Although there are so many people have the dream to start their own business, the distances between the reality and the dream are huge. In fact, only a few people into action. The main reason for this is the low rate of successful college students' entrepreneurship.

There are many factors leading to the low success rate of entrepreneurship, such as lack of capital, experience and social networks. Among these factors, how to select an appropriate project has attracted considerable attention

However, it is not an easy task to choose the most optimal entrepreneurial project from the many seemingly attractive entrepreneurial projects. There are a lot of factors need to be considered, such as the entrepreneurial project risk, the difficulty of financing, the market conditions, the profitability of the entrepreneurial project, the support of family and friends, the policy environment. What's more, it is hard to know the exactly relative importance of these factors. The existing research methodologies haven't fully solved this problem.

In this paper, we propose the SMAA-based method. This method can evaluate all of the optional entrepreneurial projects and find the most appropriate entrepreneurial projects even without knowing the relative importance of the evaluation criterion. 
This study is structured as follows. In the second section, we briefly review the previous literatures. In the third section, we propose the SMAA-based method. The conclusion of this research is in the last section.

\section{Literature review}

The entrepreneurship research began in the middle and late 20th century. In 1947, professor of Myles Mace taught the first known entrepreneurship course at Harvard University. This may be the earliest systematic research activity about the entrepreneurship [3]. However, entrepreneurship research has stagnated in a long period since then. Until the 1960s, the rapid development of the silicon semiconductor technology has spawned a large number of new businesses, which triggered the research enthusiasm of entrepreneurship. More and more investigations on entrepreneurship are made by scholars. So far, there are three main topics in the domain of entrepreneurship.

The first topic is the study of characteristics of entrepreneurs [12]. As we all know, the entrepreneurs is the core of the entrepreneurial activity. Entrepreneurs can be individuals, teams, or an organization, etc. The current study attempts to explore the basic function, the behavioral characteristics and the personality traits of the entrepreneurs from the perspective of psychology, behavioral science and sociology. [10/13]. The second topic is the entrepreneurial environment which is the sum of the internal and external factors affecting entrepreneurship in the entrepreneurial process, like the macro and micro economic environment, political environment, legal environment, cultural environment, organizational structure, team, funds and corporate culture. Many researchers have studied the impact of entrepreneurial environment for entrepreneurship [4/6]. The third topic is the study of entrepreneurial process, which including opportunities identification, business definition, resource assessment, organization creation and management of new business [1/2/5/7].

The research question of this paper is generally emerges in the entrepreneurial process of opportunity identification stage. The entrepreneurs should evaluate and compare the optional entrepreneurial projects and find the best entrepreneurial projects to start without knowing the relative importance of the evaluation criterion. However, the current research toward this problem is few and can't help the entrepreneurs perfectly.

In this paper, we focus on a multi-criteria approach for the evaluation and comparison of the entrepreneurial projects with unknown weight information about the evaluation criterion. A SMAAbased approach will be presented to capture this problem. SMAA (Stochastic multi-attribute acceptability analysis) was initially proposed by Lahdelma et al. [14] for aiding multi-attribute decision making problem with multiple alternatives by exploring the weight space. If no weight information of each evaluation index is available, SMAA assumes that all weights in the feasible weight space are equally likely to be chosen. A preferred weight subspace of each entrepreneurial project is composed of the weights making the specific alternative the most preferred one. An alternative with larger preferred weight subspace is more likely to be approved, and should be given a higher rank. All of the alternatives can be compared by measuring the area of their preferred weight subspaces. 


\section{The SMAA-based approach}

In this section, we will introduce the SMAA-based approach. Consider a entrepreneur who wants to start a new venture. There are n alternative entrepreneurial projects. Let $A=\left\{a_{1}, a_{2}, \ldots, a_{m}\right\}$ denotes the set of $\mathrm{m}$ alternatives. There are $\mathrm{n}$ criteria to evaluate each alternative entrepreneurial project. Let stochastic variables $\xi_{i}^{j}$ with joint probability distribution and density function $f(\xi)$ in the space $X$ denote the uncertain or imprecise criteria values for alternative $a_{i}$ based on criterion $j$.

Next, we will use a four-step approach to help the entrepreneur find out the optimal entrepreneurial project.

Step1. Identify the entrepreneurial projects which meet the minimum requirements. In this step, we shall set the basic requirements of criteria. The entrepreneur will abandon an entrepreneurial project if the project does not meet all of the minimum requirements. Suppose that the minimum requirement of criterion $j$ is $\xi_{j}^{0}$. Let $A_{f}$ denotes the set of feasible alternative entrepreneurial projects. Then,

$$
A_{f}=\left\{a_{i} \in A: E\left(\xi_{i}^{j}\right) \geq \xi_{j}^{0}, \text { for } j=1 \text { to } \mathrm{m}\right\}
$$

Step 2: Map the criteria value distributions into utility distribution. Next, we will select the optimal entrepreneurial projects from $A_{f}$. Some partial utility functions $u_{j}($.) to map the criterion value distributions into a utility distribution. Then, similar with the traditional multi-attribute weighting model [8/9], the overall utility $u\left(a_{i}, w\right)$ of alternative entrepreneurial project $a_{i}$ can be expressed as convex combination of the utility distributions of each criterion using some unknown normalized weights.

$$
u\left(a_{i}, w\right)=\sum_{j=1}^{n} u_{j}\left(\xi_{i}^{j}\right) w_{j}, w \in W
$$

The unknown preferences are represented by a weight distribution with joint density function $f(w)$ in the feasible space. If the preference information is totally lacked, the feasible weight space will be a space with a uniform weight distribution. The joint density function is $f(w)=1 / \operatorname{vol}(W)[9]$. The weight space can be expressed as :

$$
W=\left\{w_{1}+w_{2}+\ldots+w_{n}=1\right\}
$$

In fact, it is indeed possible that the entrepreneur has more or less partial preference information. The common partial preference may be exact numerical values of some weights, ranking of relatively importance for some criteria, and interval constraints of relatively importance for some criteria. Let $W_{P}$ denote the new weight space involves the partial preference information.

Step 3: Identify the better feasible alternative entrepreneurial project based on the partial weight information. For any two feasible alternative entrepreneurial project $a_{i} a, a^{\prime}$, it is interesting to know which one is better in view of weight information ${ }_{P}$.It is possible that if the overall expect utility value of $a$ is larger than or equal to that of $a^{\prime}$, then we say project $a$ is better than $a^{\prime}$.

Definition 2. Let $a, a^{\prime} \in A_{f}$ 。 Alternative entrepreneurial project $a$ is better than ${ }^{\prime}$ with regard to the partial weight information ${ }^{W_{P}}$, if: 


$$
E(u(a, w)) \geq E\left(u\left(a^{\prime}, w\right)\right) \text { for all } w \in W_{P}
$$

Then we delete $a^{\prime}$. Let $A_{r}$ define the rest alternative entrepreneurial project set. Then we use the SMAA-2 method to evaluate them.

Step 4: Evaluate the rest of alternative entrepreneurial projects by SMAA-2 method. Based on SMAA-2 [11] method, the rank of alternative entrepreneurial project $a_{i}$ can be calculated as an integer from the best rank to the worst rank by

$$
\operatorname{rank}\left(a_{i}, w\right)=1+\sum_{a_{i} \neq a_{j}} \rho\left(U\left(a_{i}, w\right)>U\left(a_{j}, w\right)\right)
$$

where $\rho($ true $)=1$ and $\rho($ false $)=0$. The favorable weight set for entrepreneurial project $a_{i}$ to gain rank $q$ is defined as:

$$
W_{a_{i}}^{q}=\left\{w \in W_{p}: \operatorname{rank}\left(a_{i}, w\right)=q\right\}
$$

Next we use the three evaluation tools of SMAA-2 to evaluate the rest alternative entrepreneurial project set. The five evaluation tools are proposed in the following section.

1) Rank Acceptability Index. The rank acceptability index of alternative entrepreneurial project $a_{i}$ to obtain rank $q$ is computed as a multidimensional integral over the attribute distributions and the favorable rank weights using

$$
b_{a_{i}}^{q}=\int_{\xi} f(\xi) \int_{w \in W_{a_{i}}^{q}} f_{W}(w) d w d \xi
$$

The rank acceptability index can be seen as the probability for entrepreneurial project $a_{i}$ to get rank $q$ if no weight information is given.

2) Confidence Factor. The confidence factor $p_{p_{k}}^{q}$ is defined as the probability alternative entrepreneurial project $a_{i}$ to be among the $\mathrm{k}$ best ones when the central kbr weight vector is used. The confidence factor is computed as follows.

$$
p_{a_{i}}^{q}=\int_{\xi \in X: \operatorname{rank}\left(a_{i}, \xi, w_{a_{i}}^{q}\right) \leq q} f_{x}(\xi) d \xi
$$

The confidence factor is used for judging whether the criteria measurements are accurate or not. The alternative entrepreneurial project with low confidence factors should not be chosen though the entrepreneurial project has high value of acceptability.

3) Holistic Acceptability Index. The overall acceptability evaluation index $a_{a_{i}}^{h}$ for alternative entrepreneurial project $a_{i}$ is the holistic acceptability index which is aggregated by every rank acceptability index using meta-weights $\alpha^{r}$. The holistic acceptability index is defined as

$$
a_{a_{i}}^{h}=\sum_{q} \alpha^{q} b_{a_{i}}^{q}
$$

Parameters $\alpha^{r}$ are called meta-weights which are nonnegative, normalized, and non-increasing when the rank increases.

We can use the above three tools of SMAA-2 to find out the best entrepreneurial project. In general, we can use the rank acceptability index to estimate the probabilities of each candidate entrepreneurial project to get rank $\mathrm{r}$. We use the confidence factor to eliminate those projects with low accuracy of attribute measurement. The holistic acceptability index can give the entrepreneur the overall evaluation of each project.

\section{Conclusion.}

We have presented the four-step SMAA-based approach to capture the enterprise-selection problem in the background of Chinese college venture. Each alternative entrepreneurial project is evaluated 
based on some criteria with unknown information about their relative importance. The approach is very useful when the entrepreneurs in situations where little or no weight information about the criteria is available, and the criteria values of each seemingly attractive entrepreneurial project may be inaccurate.

In this paper, we only consider the single period entrepreneurship selection problem in the environment of Chinese college student venture. The future research toward this topic may concern the muti-period entrepreneurship problem and the background of the research may be expanded to larger range like different country and different group of people.

\section{Acknowledgments.}

The financial supports from National Natural Science Foundation of China (Grant Nos. 71301039, 71322101 and 71271195) are acknowledged.

\section{References}

[1] Alexander Ardichvili, Richard Cardozo, Sourav Ray. A theory of entrepreneurial opportunity identification and development. Journal of Business Venturing 18 (2003) 105 - 123.

[2] Bhave, M., 1994. A process model of entrepreneurial venture creation. J. Bus. Venturing 9 (2), $223-242$.

[3] Brush, C.G., Duhaime, I.M., Gartner, W.B., Stewart, A., Katz, J.A., Hitt, M.A., Alvarez, S.A.,

[4] Chirstian Bruyat, Pierre-Andre Julien. Defining the field of research in entrepreneurship[J]. Journal of Business Venturing, 2001, 16(2): 165-180.

[5] De Koning, A., 1999. Conceptualizing Opportunity Recognition as a Socio-Cognitive Process. Centre for Ad-vanced Studies in Leadership, Stockholm.

[6] Devi R. Gnyawali, Daniel S. Fogel. Environments for Entrepreneurship Development: Key Dimensions and Research implications [J]. Entrepreneurship:Theory and Practice, 1994, 18(4):4362.

[7] Gartner, W B. A conceptual framework for describing the phenomenon of new venture creation[J]. Academy of Management Review, 1985, 10(4): 696-706.

[8] Golabi, K., Kirkwood, C.W., Sicherman, A.,( 1981). Selecting a portfolio of solar energy projects using multiattribute preference theory. Management Science 27, 174-189.

[9] Golabi, K., (1987), 'Selecting a group of dissimilar projects for funding'. IEEE Transactions on Engineering Management 34, 138-145.

[10] Kirzner, I. M., 1979, Perception, Opportunity and Profit: Studies in the Theory of Entrepreneurship, Chicago: University of Chicago Pres.

[11] Lahdelma, R, Salminen(2001), 'SMAA-2: Stochastic multiattribute acceptability analysis for group decision making', Operations Research 49 (3), pp444-454. 
[12] Meyer, G.D. and Venkataraman, S. (2003), "Doctoral education in the field of entrepreneurship", Journal of Management, Vol. 29 No. 3, pp. 309-31.

[13] Sternberg, R. J. 2004. Successful intelligence as a basis for entrepreneurship. Journal of Business Venturing ,2004,19(2): 189-202.

[14] Risto Lahdelma, Joonas Hokkanen, Pekka Salminen(1998), 'SMAA - Stochastic multiobjective acceptability analysis', European Journal of Operational Research, 106(1) 137-143 